\title{
Atherogenic index of plasma is associated with the severity of Hidradenitis Suppurativa: a case-control study
}

José L. Hernández ${ }^{1}$, Cristina Baldeón ${ }^{2}$ Ana E. López-Sundh³ ${ }^{3}$ J. Gonzalo Ocejo-Vinyals", Ricardo Blanco ${ }^{5}$ and Marcos A. González-López ${ }^{6^{*}}$ (D)

\begin{abstract}
Background: Hidradenitis suppurativa (HS) is a chronic inflammatory disease associated with several comorbidities and vascular risk factors, such as dyslipidemia. The present study aimed to assess the possible associations between the lipid profile and atherogenic indexes and the severity of HS.

Methods: This case-control study enrolled 78 HS patients and 62 healthy controls. Classic lipid profile and lipoprotein ratios, including the atherogenic index of plasma (AIP), were evaluated. The severity of HS was measured by the HS Physician Global Assessment (PGA).

Results: HS-patients had lower serum total cholesterol and HDL-C levels and higher AIP than the control group. AIP was positively correlated to BMI, waist circumference, systolic and diastolic blood pressure, LDL-C, triglycerides, nonHDL-C, ApoB, HOMA, and hs-CRP and negatively to HDL-C and ApoA1. For the overall lipid profile, only AIP was related to a more severe $H S(P G A \geq 3)$ after controlling for age, sex, BMI, insulin resistance (IR), active smoking, and statin use $(r=0.268 ; p=0.023)$. Multiple logistic regression adjusted for age, sex, BMI, IR, smoking status and statin use, showed that AIP $\geq 0.11$ was significantly associated with the severity of $\mathrm{HS}(\mathrm{OR}, 4.38 ; \mathrm{Cl} 95 \%, 1.09-17.50 ; p=0.037)$.
\end{abstract}

Conclusions: In conclusion, these results showed that AIP is significantly and independently associated with HS severity.

Keywords: Hidradenitis suppurativa, Atherogenic indexes, Atherogenic index of plasma, Lipids

\section{Background}

Hidradenitis suppurativa (HS) is a recurrent chronic inflammatory disease presenting with painful, suppurating lesions affecting the apocrine gland-rich regions of the skin. Prevalence is estimated from 0.05 to $4 \%$ across series, and this condition is associated with severe impairment of the quality of life [1]. HS has been related to certain comorbidities and metabolic disorders such as type 2 diabetes mellitus (T2DM) and metabolic syndrome, both

\footnotetext{
* Correspondence: marcosg@aedv.es

${ }^{6}$ Division of Dermatology, Hospital Universitario Marqués de Valdecilla, IDIV AL, University of Cantabria, 39008 Santander, Spain

Full list of author information is available at the end of the article
}

characterized by the common link of insulin resistance (IR) [2-4]. Furthermore, an increased prevalence of vascular risk factors, subclinical atherosclerosis, and a significant risk for major adverse cardiovascular events have also been reported in HS-patients [5-7].

The precise inflammatory mechanism underlying HS is still not fully elucidated, although genetic susceptibility, mechanical stress, obesity, microbiome, and environmental and hormonal factors have been involved $[8,9]$. Moreover, in HS-patients, the presence in the affected skin areas and/or in the serum of increased expression of several cytokines, such as interleukin (IL)-1 $\beta$, IL-6, IL17, interferon-gamma, and tumor necrosis factor-alpha 
(TNF- $\alpha$ ) suggest a role for the autoinflammation in HS pathogenesis [9].

Atherogenic dyslipidemia, i.e. the combined occurrence of high fasting blood concentrations of triglycerides (TG) and low levels of high-density lipoprotein cholesterol (HDL-C), is frequent in patients with HS [10]. Nevertheless, data on the relationship between the values of nontraditional lipid profiles (lipoprotein ratios), including non-HDL-C, total cholesterol (TC)/HDL-C, LDL-C/HDL-C, non-HDL-C/HDL-C (atherogenic index, $\mathrm{AI})$, atherogenic index of plasma (AIP) and Apolipoprotein $\mathrm{B} / \mathrm{Apolipoprotein} \mathrm{A} 1$ (ApoB/ApoA1) ratio, and the severity of HS are lacking. Compared with single lipid parameters, these comprehensive lipid ratios are considered to be better predictors for coronary artery disease $[11,12]$, mainly the AIP [13-15]. This index has shown a good correlation with smaller LDL-C particles and also with increased fractional esterification rate for cholesterol in plasma, and it is a strong and independent predictor factor for coronary disease [16-18]. Noteworthy, AIP has also been associated with raised serum Creactive protein (CRP) levels, suggesting a lipid-driven immune-inflammatory link [19].

Taking into account the above considerations, the present study aimed to assess the possible associations between the lipid profile and atherogenic indexes and the severity of HS.

\section{Methods}

\section{Study population}

In this cross-sectional case-control study, 78 patients with HS and 62 age- and gender-similar controls were included. HS-patients were recruited from the Dermatology outpatient clinic of a teaching tertiary-care hospital in Northern Spain. The control group was set up with hospital medical staff and subjects who attended the Dermatology clinic due to skin disorders other than HS, such as epithelioma, warts, or melanocytic nevus. The flow-chart of the study subjects is shown in Fig. 1. The research protocol was approved by the local Ethics Committee (internal code: 2013.267) and all study procedures were done under the ethical principles of the Declaration of Helsinki. All the participants provided written informed consent.

For the present study, exclusion criteria were as follows: age $<18$ years, documented history of major adverse cardiovascular events, chronic kidney or liver diseases, and the presence of concomitant inflammatory cutaneous or systemic disorders.

\section{Data collection and working definitions}

The following variables were collected in all the participants: demographic features, past medical history, current and prior systemic therapy, traditional

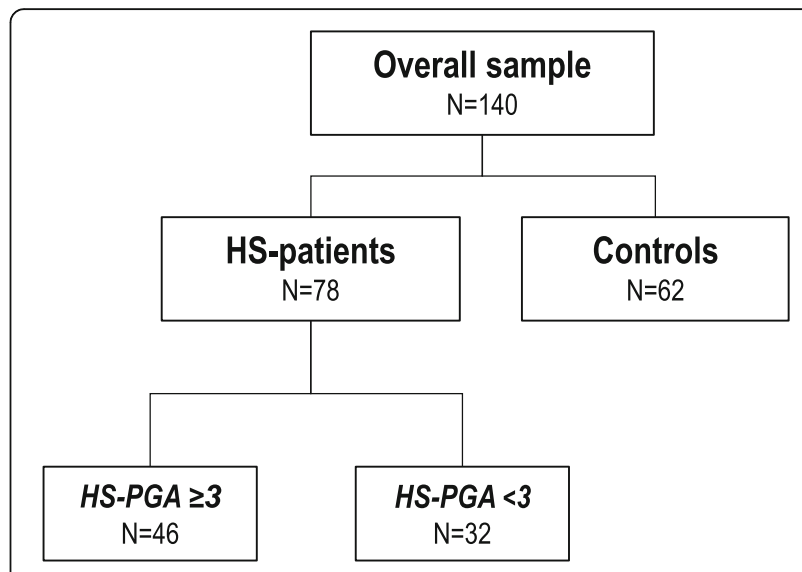

Fig. 1 Flow chart of the study subjects. Footnote: HS-PGA: Hidradenitis Suppurativa Physician Global Assessment

cardiovascular risk factors (defined as previously reported) [2], weight, height, body mass index (BMI), waist circumference (WC), systolic blood pressure (BP) and diastolic BP. Information about HS duration was collected in all the patients.

The HS Physician Global Assessment (HS-PGA) and the Hurley scale were used to assessing the severity of HS. Thus, HS was classified as moderate-severe-very severe $(\mathrm{PGA} \geq 3)$ and as minimal-mild $\mathrm{HS}(\mathrm{PGA}<3)$ according to HS-PGA [2].

Metabolic syndrome was diagnosed according to the criteria proposed by the National Cholesterol Education Program's Adult Treatment Panel III (ATP III) [20]. AIP is defined as the base 10 logarithm of the ratio of the concentration of TG to HDL-C with TG and HDL-C expressed in $\mathrm{mmol} / \mathrm{L}$ [14].

\section{Laboratory assessments}

Blood samples were drawn after an overnight fast, and serum TC, LDL-C, HDL-C, TG, ApoB, ApoA1 and lipoprotein (a) (Lpa) were analyzed, as well as the following atherogenic indexes: TC/HDL-C; LDL-C/HDL-C; nonHDL-C/HDL-C; ApoB/ApoA1, and AIP. Moreover, the homeostatic model assessment for IR (HOMA-IR) was calculated as fasting insulin level $(\mu \mathrm{IU} / \mathrm{ml}) \mathrm{x}$ fasting glucose level $(\mathrm{mg} / \mathrm{dL}) / 405$. A value greater than 2.5 is consistent with IR [2].

\section{Statistical analysis}

Results were expressed as numbers (percentage), mean \pm standard deviation (SD) or median and interquartile range (IQR), as appropriate. Student's T-test or MannWhitney U-test was used to compare quantitative variables and Chi-squared or Fisher test, to compare qualitative data, as appropriate. The Spearman correlations coefficients were calculated to assess the relationship between AIP and demographic and laboratory parameters 
in cases and controls. The patients were divided into two groups according to disease severity based on the PGA score $(<3$ and $\geq 3)$. A cut-off point for AIP $\geq 0.11$ for both sexes, based on previously published data, has been considered for the analysis [21].To assess the potential association between AIP and HS severity, forward stepwise multiple logistic regression models adjusted for confounder variables were built. The strength of the association between the study parameters and HS severity was evaluated via the odds ratio (OR) and 95\% confidence interval (CI). Finally, ROC curve analysis has been performed to test the accuracy of the regression model. A $P$-value $<0.05$ was considered significant in all the calculations.

\section{Results}

\section{Baseline features}

The mean age of HS patients and controls was $43 \pm 12$ years and $46 \pm 13$, respectively $(P=0.16)$. Nearly half of the patients and controls were male. Thirteen HS patients and 12 controls were on statins at baseline $(P=$ $0.68)$. One patient and 4 controls were taking ezetimibe $(P=0.16)$, and $2 \mathrm{HS}$ patients were on fenofibrate $(P=$ $0.16)$. There were no differences regarding the dose of statin between patients and controls $(P=0.83)$.

Patients with HS had significantly higher weight $(82.4 \pm 17.8$ vs. $76.4 \pm 16.9 \mathrm{Kg} ; P=0.001)$, BMI $(29.4 \pm 5.4$ vs. $26.5 \pm 4.5 \mathrm{Kg} / \mathrm{m} 2 ; P=0.001)$, waist circumference $(99.6 \pm 14$ vs. $91.4 \pm 14 \mathrm{~cm}: P=0.001)$, systolic blood pressure (132.6 \pm 16.4 vs. $124.2 \pm 15.8 \mathrm{mmHg} ; P=0.003)$, diastolic blood pressure $(82.0 \pm 13.8$ vs. $76.9 \pm 8.3 \mathrm{mmHg}$; $P=0.012)$, HOMA-IR values $(2.15[1.00-3.73]$ vs. 1.48 [0.89-2.28]: $P=0.005)$, high-sensitive C-reactive protein
-hs-CRP- $(0.42[0.17-0.89]$ vs. $0.10[0.10-0.20] ; P<$ $0.0001)$ and prevalence of smoking $(65.4 \%$ vs. $19.4 \% ; P<$ $0.0001)$, IR (46.2 vs. $19.4 ; p=0.001)$ and metabolic syndrome (32.7 vs. $11.9 ; P=0.004$ ), than controls. Moreover, HS-patients had lower serum total cholesterol and HDL-C levels and higher AIP than the control group (Table 1).

\section{Correlation between AIP and other variables}

The correlations between AIP and several anthropometric and laboratory parameters, in cases and controls, are shown in Table 2. Noteworthy, AIP is significantly and positively related to BMI and waist circumference, systolic and diastolic blood pressure, and several lipid parameters, but also to HOMA and hs-CRP. Correlations were negative, as expected, with serum HDL-C and ApoA1 levels.

\section{AIP and HS severity}

For the overall lipid profile analyzed, only AIP $(r=$ 0.316; $P=0.005)$ was related to a more severe HS $(P G A \geq 3)$. After controlling for age, sex, BMI, IR, statin use, and active smoking this relationship persisted significant $(r=0.268 ; P=0.023)$. No relationship between AIP and duration of HS was found. Table 3 shows the significant variables in $\mathrm{HS}$ patients according to the PGA score.

The results of multiple logistic regression analysis of the parameters with potential association with HS severity, adjusted for age, sex, BMI, IR, smoking status, and statin use are shown in Table 4. Further adjustment for the duration of HS, hypertension, serum fibrinogen levels, and hs-CRP did not change these results. The

Table 1 Lipid profile and atherogenic indexes in HS patients and controls

\begin{tabular}{llll}
\hline Parameter & HS patients $(n=78)$ & Controls $(n=62)$ & $P$ \\
\hline Total cholesterol, $m g / d L$ & $186.4 \pm 33.6$ & $202.7 \pm 44.7$ & 0.015 \\
LDL-C, $m g / d L$ & $116.6 \pm 32.4$ & $122.4 \pm 29.2$ & 0.28 \\
HDL-C, $m g / d L$ & $46.0(41.0-56.3)$ & $52.5(46.8-69.5)$ & 0.001 \\
TG, $m g / d L$ & $87.5(68.8-117.3)$ & $74.0(57.8-121.5)$ & 0.23 \\
Non-HDL cholesterol, $m g / d L$ & $138.5(115.0-155.3)$ & $136.5(118.3-160.3)$ & 0.45 \\
ApoA1, mg/dL & $146.0(127.0-167.0)$ & $151.0(134.0-173.0)$ & 0.12 \\
ApoB, mg/dL & $98.0(79.5-112.3)$ & $90.0(77.8-104.3)$ & 0.28 \\
Lpa, mg/dL & $15.0(7.6-27.5)$ & $15.5(6.0-36.0)$ & 0.83 \\
Total cholesterol / HDL-C & $4.09(3.09-4.76)$ & $3.51(2.83-4.40)$ & 0.06 \\
LDL-C / HDL-C & $2.49(1.85-3.12)$ & $2.19(1.69-3.02)$ & 0.09 \\
Non-HDL-C / HDL-C & $3.09(2.09-3.76)$ & $2.51(1.83-3.40)$ & 0.06 \\
LDL-C / ApoB & $1.22(1.09-1.29)$ & $1.35(12.5-1.45)$ & $<0.0001$ \\
ApoB / ApoA1 & $0.65(0.51-0.82)$ & $0.60(0.47-0.70)$ & 0.09 \\
AlP & $-0.07(-0.24-0.05)$ & $-0.23(-0.41-0.02)$ & 0.016 \\
\hline
\end{tabular}

$B M I$ body mass index, $L D L-C$ low-density lipoprotein, $H D L-C$ high-density lipoprotein, $T G$ triglycerides, $L p a$ lipoprotein (a), AIP atherogenic index of plasma Values are expressed as mean $\pm S D$ or median (interquartile range) as appropriate 
Table 2 Correlation between the atherogenic index of plasma and some demographic and laboratory variables in HS patients and controls

\begin{tabular}{|c|c|c|c|c|}
\hline & \multicolumn{2}{|c|}{ HS patients $(n=78)$} & \multicolumn{2}{|c|}{ Controls $(n=62)$} \\
\hline & $\bar{r}$ & $P$ & $\bar{r}$ & $P$ \\
\hline Age, years & 0.233 & 0.049 & 0.210 & 0.10 \\
\hline $\mathrm{BMI}, \mathrm{Kg} / \mathrm{m}^{2}$ & 0.352 & 0.002 & 0.332 & 0.008 \\
\hline Waist circumference, $\mathrm{cm}$ & 0.399 & $<0.0001$ & 0.349 & 0.005 \\
\hline Systolic BP, $\mathrm{mmHg}$ & 0.231 & 0.042 & 0.277 & 0.03 \\
\hline Diastolic BP, mmHg & 0.230 & 0.042 & 0.304 & 0.016 \\
\hline Total cholesterol, mg/dL & 0.035 & 0.76 & 0.187 & 0.15 \\
\hline $\mathrm{LDL}-\mathrm{C}, \mathrm{mg} / \mathrm{dL}$ & 0.136 & 0.24 & 0.266 & 0.038 \\
\hline $\mathrm{HDL}-\mathrm{C}, \mathrm{mg} / \mathrm{dL}$ & -0.655 & $<0.0001$ & -0.709 & $<0.0001$ \\
\hline $\mathrm{TG}, \mathrm{mg} / \mathrm{dL}$ & 0.884 & $<0.0001$ & 0.912 & $<0.0001$ \\
\hline Non-HDL cholesterol, mg/dL & 0.380 & 0.001 & 0.554 & $<0.0001$ \\
\hline ApoA1, mg/dL & -0.408 & $<0.0001$ & -0.499 & $<0.0001$ \\
\hline ApoB, mg/dL & 0.331 & 0.003 & 0.587 & $<0.0001$ \\
\hline $\mathrm{Lpa}, \mathrm{mg} / \mathrm{dL}$ & -0.080 & 0.48 & -0.141 & 0.27 \\
\hline Fasting glucose, mg/dL & 0.258 & 0.023 & 0.128 & 0.32 \\
\hline $\mathrm{hs}-\mathrm{CRP}, \mathrm{mg} / \mathrm{dL}$ & 0.343 & 0.002 & 0.362 & 0.004 \\
\hline $\mathrm{HbA} 1 \mathrm{c}, \%$ & 0.375 & 0.001 & 0.181 & 0.16 \\
\hline HOMA index & 0.551 & $<0.0001$ & 0.400 & 0.001 \\
\hline Insulin, $m / U / L$ & 0.491 & $<0.0001$ & 0.370 & 0.003 \\
\hline
\end{tabular}

$B M I$ body mass index, $B P$ blood pressure, $L D L-C$ low-density lipoprotein, $H D L-C$ high-density lipoprotein, TG triglycerides, Lpa lipoprotein (a), hs-CRP highsensitive $C$ reactive protein, HOMA homeostatic model assessment

exclusion of participants on statins (HS-patients and controls) yielded virtually identical results. Figure 2 shows the ROC curve for the regression model. The area under the curve is 0.73 (95\%CI, 0.61-0.84; $P=0.001)$.

\section{Discussion}

Lipids are biological compounds that play multiple roles in human disease. Thus, neutral lipids, such as TG are critical in energy storage and have been involved in the

Table 3 Significant variables in HS patients according to the PGA score

\begin{tabular}{llll}
\hline Parameter & $\mathrm{PGA}<3(n=32)$ & $\mathrm{PGA} \geq 3(n=46)$ & $P$ \\
\hline Age, years & $39.1 \pm 11.6$ & $45.1 \pm 11.2$ & 0.023 \\
BMI, Kg/m2 & $28.0 \pm 5.5$ & $30.4 \pm 5.2$ & 0.043 \\
Duration of HS, months & $13.5(5.3-22.5)$ & $19.0(9.8-27.3)$ & 0.039 \\
TG, $\mathrm{mg} / \mathrm{dL}$ & $71.0(53.0-103.5)$ & $93.0(75.5-143.3)$ & 0.003 \\
AIP & $-0.19(-0.43-0.02)$ & $-0.04(-0.17-0.18)$ & 0.006 \\
hs-CRP, mg/dL & $0.23(0.12-0.58)$ & $0.57(0.31-1.06)$ & 0.003 \\
Fibrinogen, $\mathrm{mg} / \mathrm{dL}$ & $281.0(264.5-324.3)$ & $338.0(289.0-392.8)$ & 0.007 \\
\hline
\end{tabular}

$B M I$ body mass index, LDL-C low-density lipoprotein, $H D L-C$ high-density lipoprotein, TG triglycerides, Lpa lipoprotein (a), hs-CRP high-sensitive $C$ reactive protein, AIP atherogenic index of plasma

Values are expressed as mean $\pm S D$ or median (interquartile range) as appropriate
Table 4 Adjusted multiple logistic regression analysis showing the best set of factors associated with HS severity

\begin{tabular}{llll}
\hline & $\beta$-coefficient & OR $(95 \% C l)$ & $P$ \\
\hline Age, years & 0.050 & $1.05(1.005-1.10)$ & 0.03 \\
AlP $\geq 0.11$ & 1.476 & $4.38(1.09-17.50)$ & 0.037 \\
\hline AIP atherogenic index of plasma & &
\end{tabular}

pathogenesis of cardiovascular diseases, metabolic syndrome, and T2DM [22]. Lipid signaling pathways in patients with HS are poorly understood as well as the cellular mechanisms of lipid-mediated HS-induced pathogenesis. Gene expression of certain sphingolipids such as ceramide and sphingosine-1, that act as biologically active signaling molecules, have been implicated in the pathogenesis of the disease [23]. In a recent report, Fincher et al. [24], showed an increased localized accumulation of neutral lipids in HS-infected tissue as a result of a great bacterial load in these lesions. Moreover, recurrence of the disease has been linked to the development of dermic and subcutaneous sinus tracts, and lipids rafts in plasma membranes of keratinocytes also play a role in the regulation of metabolic and proliferative activity of these cells in HS patients [25]. Local steroidogenic activities in the skin have been implicated in the regulation of immune responses at local or systemic levels, and impaired of this cutaneous steroidogenesis has been linked to inflammatory skin disorders [26]. Finally, recent studies have shown that proprotein convertase subtilisin/kexin type 9 (PCSK9), a key regulator of lipid metabolism, may have pro-inflammatory activity on macrophages and has been linked to some markers of systemic inflammation, such as hs-CRP [27]. Nevertheless, there is no evidence to date, on its involvement in HS pathogenesis.

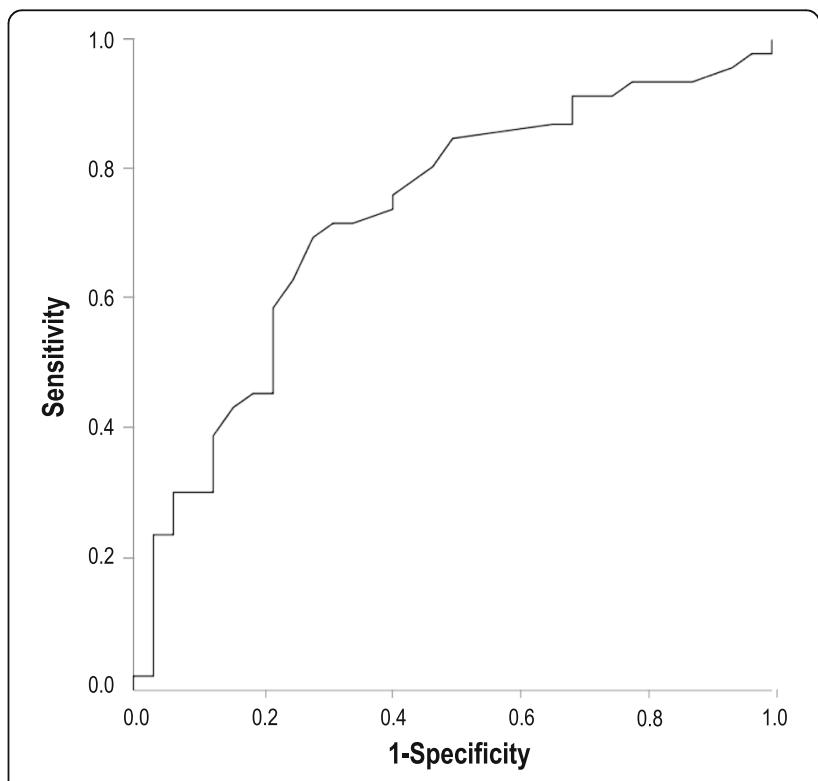

Fig. 2 ROC curve for the regression model 
In clinical studies, HS patients often have higher serum TG and lower HDL-C levels than controls. The present study confirms a significant decrease in HDL-C in patients with HS compared with healthy controls. In this sense, Tsaousi et al. [28], found that matrix metalloproteinase 8 , a collagen cleaving enzyme involved in the breakdown of extracellular matrix in normal and pathological processes, and in the degradation of ApoA1, a component of HDL particles, is one of the most highly upregulated molecules in HS lesions.

More recently, the AIP value, a logarithmically transformed ratio of molar concentrations of TG to HDL-C, has been reported as a good marker for the risk of atherosclerosis and cardiovascular disease [29]. AIP is an easily calculated parameter from the standard lipid profile that adds predictive value beyond that of the individual lipids and/or TC/HDL-C ratio. Furthermore, it is considered a subrogate of small LDL-C particle size distribution, with a better correlation than the LDL-C/ ApoB ratio [30]. AIP provides additional information in predicting short- and long-term outcomes in patients with acute coronary syndrome but also it may be an independent factor for the risk of type 2 diabetes mellitus and metabolic syndrome [31, 32].

Interestingly, in HS patients (even after excluding the few participants on lipid-lowering agents) serum LDL-C, the traditional marker of the atherosclerotic burden was similar to controls. Nevertheless, AIP was higher in patients with HS than controls. In this sense, since HS is associated with high cardiovascular morbidity, AIP could be used as a good predictor of cardiovascular risk in these patients even in the presence of a normal lipid profile. Further studies on this matter would be interesting to perform.

In this study, AIP is related to BMI, waist circumference, blood pressure, lipid parameters, hs-CRP, and insulin resistance. Nevertheless, in the present study, AIP is an independent factor for the risk of a more severe HS, measured by the PGA. Besides, this association seems to be independent of hs-CRP, a well-known marker of inflammation. Besides, a cut-off point of 0.11 for this index has demonstrated to have a 4-fold increased risk for a PGA score $\geq 3$. This index could be useful not only to detect patients at high risk for metabolic (obesity, diabetes, or metabolic syndrome) or cardiovascular complications (high blood pressure, cardiovascular events) but also to alert the clinician to the presence of a more severe HS.

\section{Study strength and limitations}

This is the first study assessing the role of AIP in patients with HS. Nevertheless, it has the inherent limitations of a case-control study regarding causality. Besides, as an observational study, it may be subject to some bias due to the possible existence of confounders. However, to try to avoid this issue, adjustment for multiple potential confounding factors has been carried out.

\section{Conclusions}

AIP is significantly and independently associated with a more severe HS. Therefore, clinicians might consider this index in the overall assessment of HS patients. Further large prospective studies are needed to confirm these results and explore the underlying mechanisms of the lipid-mediated HS-induced pathogenesis.

\section{Abbreviations}

AIP: Atherogenic Index of Plasma; ApoA1: Apolipoprotein A1; ApoB: Apolipoprotein B100; BMI: Body Mass Index; BP: Blood Pressure; HDLC: High-density Lipoprotein Cholesterol; HOMA: Homeostatic Model Assessment; HS: Hidradenitis Suppurativa; hs-CRP: High-sensitive C-Reactive Protein; IR: Insulin Resistance; LDL-C: Low-density Lipoprotein Cholesterol; Lpa: Lipoprotein (a); PGA: Physician Global Assessment; PCSK9: Proprotein convertase subtilisin/kexin type 9; T2DM: Type 2 Diabetes Mellitus; TC: Total Cholesterol; TG: Triglycerides; TNF-a: Tumor Necrosis Factor-alpha; WC: Waist Circumference

\section{Acknowledgements}

Not applicable.

\section{Authors' contributions}

JLH performed the study, contributed to the elaboration of the protocol of the study, helped in the interpretation of the data, and was responsible for the final drafting and elaboration of the manuscript. CB performed the study, helped in the interpretation of data, and the elaboration of the manuscript. AEL-S, GO-V, and RB helped in the interpretation of data and the elaboration of the manuscript. MAG-L recruited the patients for the study, contributed to the elaboration of the protocol of the study, performed the study, helped in the interpretation of data, and was responsible for the final drafting and elaboration of the manuscript. The author(s) read and approved the final manuscript.

\section{Funding}

None.

\section{Availability of data and materials}

The datasets generated and/or analyzed during the current study are not publicly available due to the protection of patients' identity but are available from the corresponding author on reasonable request.

\section{Ethics approval and consent to participate}

This study was approved by the Comité de Ética de Investigación Clínica (CEIC) in Cantabria (internal code: 2013.267). Written informed consent was obtained from each patient included in the study.

\section{Consent for publication}

Not applicable.

\section{Competing interests}

Dr. Hernandez reports research grants from Amgen, and fees for participation in company-sponsored speaker's bureau from Amgen, MSD, and Bayer. Dr. R. Blanco received grants/research supports from Abbvie, MSD, and Roche, and had consultation fees/participation in company-sponsored speaker's bureau from Abbvie, Pfizer, Roche, Bristol Myers, Janssen, and MSD. Dr. González-López had consultation fees/participation in companysponsored speaker's bureau from Abbvie. The rest of the authors declare that they have no competing interests.

\section{Author details}

'Division of Internal Medicine, Hospital Universitario Marqués de Valdecilla, University of Cantabria, IDIVAL, Santander, Spain. ${ }^{2}$ Division of Internal Medicine, Hospital Universitario Marqués de Valdecilla, University of 
Cantabria, Santander, Spain. ${ }^{3}$ Division of Dermatology, Hospital Universitario Marqués de Valdecilla, University of Cantabria, Santander, Spain. ${ }^{4}$ Division of Immunology, Hospital Universitario Marqués de Valdecilla, University of Cantabria, IDIVAL, Santander, Spain. ${ }^{5}$ Division of Rheumatology, Hospital Universitario Marqués de Valdecilla, University of Cantabria, IDIVAL, Santander, Spain. ${ }^{6}$ Division of Dermatology, Hospital Universitario Marqués de Valdecilla, IDIVAL, University of Cantabria, 39008 Santander, Spain.

Received: 10 July 2020 Accepted: 26 August 2020

Published online: 29 August 2020

\section{References}

1. Zouboulis CC, Desai N, Emtestam L, Hunger RE, loannides D, Juhász I, et al. European $\mathrm{S} 1$ guideline for the treatment of hidradenitis suppurativa/acne inversa. J Eur Acad Dermatol Venereol. 2015;29:619-44.

2. Vilanova I, Hernández JL, Mata C, Duran C, García-Unzueta MT, Portilla V, et al. Insulin resistance in hidradenitis suppurativa: a case-control study. Eur Acad Dermatol Venereol. 2018;32:820-4.

3. Ergun T. Hidradenitis suppurativa and the metabolic syndrome. Clin Dermatol. 2018:36:41-7.

4. Phan K, Charlton O, Smith SD. Hidradenitis suppurativa and diabetes mellitus: updated systematic review and adjusted meta-analysis. Clin Exp Dermatol. 2019;44:e126-32.

5. González-López MA, Hernández JL, Lacalle M, Mata C, López-Escobar M, López-Mejías R, et al. Increased prevalence of subclinical atherosclerosis in patients with hidradenitis suppurativa. J Am Acad Dermatol. 2016;75:329-35.

6. Egeberg A, Gislason GH, Hansen PR. Risk of major adverse cardiovascular events and all-cause mortality in patients with hidradenitis suppurativa. JAMA Dermatol. 2016;152:429-34

7. Gremese E, Tolusso B, Gigante MR, Ferraccioli G. Obesity as a risk and severity factor in rheumatic diseases (autoimmune chronic inflammatory diseases). Front Immunol. 2014;11:576.

8. Negus D, Ahn C, Huang W. An update on the pathogenesis of hidradenitis suppurativa: implications for therapy. Expert Rev Clin Immunol. 2018;14:27583.

9. Vossen ARJV, van der Zee HH, Prens EP. Hidradenitis Suppurativa: A Systematic Review Integrating Inflammatory Pathways Into a Cohesive Pathogenic Model. Front Immunol. 2018;14(9):2965.

10. Tzellos T, Zouboulis CC, Gulliver W, Cohen AD, Wolkenstein P, Jemec GBE. Cardiovascular disease risk factors in patients with hidradenitis suppurativa: a systematic review and meta-analysis of observational studies. $\mathrm{Br} J$ Dermatol. 2015;173:1142-55.

11. Gao M, Zheng Y, Zhang W, Cheng Y, Wang L, Qin L. Non-high-density lipoprotein cholesterol predicts nonfatal recurrent myocardial infarction in patients with ST-segment elevation myocardial infarction. Lipids Health Dis. 2017;16:20.

12. Zhu L, Lu Z, Zhu L, Ouyang $X$, Yang $Y$, He W, et al. Lipoprotein ratios are better than conventional lipid parameters in predicting coronary heart disease in Chinese Han people. Kardiol Pol. 2015;73:931-8.

13. Niroumand $\mathrm{S}$, Khajedaluee $\mathrm{M}$, Khadem-Rezaiyan $\mathrm{M}$, Abrishami M, Juya $\mathrm{M}$, Khodaee G, et al. Atherogenic index of plasma (AIP): a marker of cardiovascular disease. Med J Islam Repub Iran. 2015;29:240.

14. Onat A, Can G, Kaya H, Hergenç G. "Atherogenic index of plasma" ( $\log 10$ triglyceride/high-density lipoprotein cholesterol) predicts high blood pressure, diabetes, and vascular events. J Clin Lipidol. 2010;4:89-98.

15. Zhan $Y, X u T$, Tan $X$. Two parameters reflect lipid-driven inflammatory state in acute coronary syndrome: atherogenic index of plasma, neutrophillymphocyte ratio. BMC Cardiovasc Disord. 2016;16:96.

16. Dobiasova M, Frohlich J. The plasma parameter log (TG/HDL-C) as an atherogenic index: correlation with lipoprotein particle size and esterification rate in apoB-lipoprotein-depleted plasma (FER(HDL)). Clin Biochem. 2001;34:583-8.

17. Frohlich J, Dobiasova M. Fractional esterification rate of cholesterol and ratio of triglycerides to HDL-cholesterol are powerful predictors of positive findings on coronary angiography. Clin Chem. 2003;49:1873-80.

18. Cai G, Shi G, Xue S, Lu W. The atherogenic index of plasma is a strong and independent predictor for coronary artery disease in the Chinese Han population. Medicine (Baltimore). 2017;96:e8058.

19. Falk E, Nakano M, Bentzon JF, Finn AV, Virmani R. Update on acute coronary syndromes: the pathologists' view. Eur Heart J. 2013;34:719-28.
20. National Cholesterol Education Program (NCEP) Expert Panel on Detection. Evaluation, and treatment of high blood cholesterol in adults (adult treatment panel III) third report of the National Cholesterol Education Program (NCEP) expert panel on detection, evaluation, and treatment of high blood cholesterol in adults (adult treatment panel III) final report (2002). Circulation. 2002;106:3143-421.

21. Ahn SS, Lee LE, Pyo JY, Song JJ, Park YB, Lee SW. Atherogenic index of plasma predicts cerebrovascular accident occurrence in antineutrophil cytoplasmic antibody-associated vasculitis. Lipids Health Dis. 2020;19:184.

22. Hannun YA, Obeid LM. Principles of bioactive lipid signaling: lessons from sphingolipids. Nat Rev Mol Cell Biol. 2008;9:139-50.

23. Dany $\mathrm{M}$, Elston $\mathrm{D}$. Gene expression of sphingolipid metabolism pathways is altered in hidradenitis suppurativa. J Am Acad Dermatol. 2017;77:268-73.

24. Fincher JA, Jones DR, Korte AR, Dyer JE, Parlanti P, Popratiloff A, et al. Mass spectrometry imaging of lipids in human skin disease model Hidradenitis Suppurativa by laser desorption ionization from silicon Nanopost arrays. Sci Rep. 2019;9:17508.

25. Gniadecki R, Jemec GB. Lipid raft-enriched stem cell-like keratinocytes in the epidermis, hair follicles and sinus tracts in hidradenitis suppurativa. Exp Dermatol. 2004;13:361-3.

26. Slominski A, Zbytek B, Nikolakis G, Manna PR, Skobowiat C, Zmijewski M, et al. Steroidogenesis in the skin: implications for local immune functions. J Steroid Biochem Mol Biol. 2013;137:107-23.

27. Cesaro A, Bianconi V, Gragnano F, Moscarella E, Fimiani F, Monda E, et al. Beyond cholesterol metabolism: the pleiotropic effects of proprotein convertase subtilisin/kexin type 9 (PCSK9). Genetics, mutations, expression, and perspective for long-term inhibition. Biofactors. 2020;46:367-80.

28. Tsaousi A, Witte E, Witte K, Röwert-Huber HS, Volk HD, Sterry W, et al. MMP8 is increased in lesions and blood of acne Inversa patients: a potential link to skin destruction and metabolic alterations. Mediat Inflamm. 2016;2016: 4097574.

29. Dobiásová M, Frohlich J, Sedová M, Cheung MC, Brown BG. Cholesterol esterification and atherogenic index of plasma correlate with lipoprotein size and findings on coronary angiography. J Lipid Res. 2011;52:566-71.

30. Furuya D, Yagihashi A, Nasu S, Endoh T, Nakamura T, Kaneko R, et al. LDL particle size by gradient-gel electrophoresis cannot be estimated by LDLcholesterol/apolipoprotein B ratios. Clin Chem. 2000;46(8 Pt 1):1202-3.

31. Zhu XW, Deng FY, Lei SF. Meta-analysis of Atherogenic index of plasma and other lipid parameters in relation to risk of type 2 diabetes mellitus. Prim Care Diabetes. 2015;9:60-7.

32. Zhang X, Zhang X, Li X, Feng J, Chen X. Association of metabolic syndrome with atherogenic index of plasma in an urban Chinese population: a 15year prospective study. Nutr Metab Cardiovasc Dis. 2019;29:1214-9.

\section{Publisher's Note}

Springer Nature remains neutral with regard to jurisdictional claims in published maps and institutional affiliations.

Ready to submit your research? Choose BMC and benefit from:

- fast, convenient online submission

- thorough peer review by experienced researchers in your field

- rapid publication on acceptance

- support for research data, including large and complex data types

- gold Open Access which fosters wider collaboration and increased citations

- maximum visibility for your research: over $100 \mathrm{M}$ website views per year

At $\mathrm{BMC}$, research is always in progress.

Learn more biomedcentral.com/submissions 